\title{
7th Edition of the AJCC Cancer Staging Manual: Esophagus and Esophagogastric Junction
}

\author{
Thomas W. Rice, $\mathrm{MD}^{1}$, Eugene H. Blackstone, $\mathrm{MD}^{1,2}$, and Valerie W. Rusch, $\mathrm{MD}^{3}$ \\ ${ }^{1}$ Department of Thoracic and Cardiovascular Surgery, Heart and Vascular Institute, Cleveland Clinic, Cleveland, $\mathrm{OH}$; \\ ${ }^{2}$ Department of Quantitative Health Sciences, Research Institute, Cleveland Clinic, Cleveland, OH; ${ }^{3}$ Department of \\ Surgery, Thoracic Service, Memorial Sloan-Kettering Cancer Center, New York, NY
}

In previous editions of the American Joint Committee on Cancer (AJCC) Cancer Staging Manual, esophageal cancer staging was neither data driven nor harmonized with stomach cancer. The new staging system presented in the 7th edition of the AJCC Cancer Staging Manual, in contrast, is data driven and harmonized. ${ }^{1}$ This commentary describes development of the new system and highlights the changes and additions.

\section{DATA AND ANALYSIS}

At the request of the AJCC, worldwide data were assembled to develop the 7th edition staging system for esophageal cancer. ${ }^{1}$ The Worldwide Esophageal Cancer Collaboration (WECC) was inaugurated in 2006. Thirteen institutions from five countries and three continents (Asia, Europe, and North America) submitted deidentified data by July 2007. These were used to construct a database of 4,627 esophagectomy patients who had no induction or adjuvant therapy to develop a staging system based on the pathologic tumor, node, metastasis (TNM) system. ${ }^{2}$

Several previously proposed revisions of esophageal cancer staging have examined goodness of fit or $P$ values to test for a statistically significant effect of stage on survival. However, staging for the 7th edition used Random Forest (RF) analysis, a machine-learning technique that focuses on predictiveness for future patients. ${ }^{3} \mathrm{RF}$ analysis makes no a priori assumptions about patient survival, is able to identify complex interactions among variables, and accounts for nonlinear effects.

(C) Society of Surgical Oncology 2010

Published Online: 6 April 2010

T. W. Rice, MD

e-mail: ricet@ccf.org
RF analysis first isolated cancer characteristics of interest from other factors influencing survival by generating risk-adjusted survival curves for each patient. Unlike previous approaches that began by placing cancer characteristics into proposed groups, RF analysis produced distinct groups with monotonically decreasing risk-adjusted survival without regard to cancer characteristics. Then anatomic and nonanatomic cancer characteristics important for stage group composition were identified within these groups. Homogeneity within groups guided both amalgamation and segmentation of cancer characteristics between adjacent groups to arrive at the proposed stage groups. $^{4}$

The final step involved consensus panels within WECC and with the AJCC Gastric Task Force, AJCC, and International Union Against Cancer (UICC) aimed at filling certain gaps in the data, meeting AJCC and UICC definitions and requests, and harmonizing with gastric cancer staging. ${ }^{1}$

\section{CLASSIFICATIONS: CHANGES AND ADDITIONS IN THE 7TH EDITION}

$\mathrm{T}$ classification has been changed for Tis and $\mathrm{T} 4$ cancers (Table 1). Tis is now defined as high-grade dysplasia and includes all noninvasive neoplastic epithelium that was previously called carcinoma-in-situ. T4, tumors invading local structures, have been subclassified as $\mathrm{T} 4 \mathrm{a}$ and $\mathrm{T} 4 \mathrm{~b}$. $\mathrm{T} 4 \mathrm{a}$ tumors are resectable cancers invading adjacent structures such as pleura, pericardium, or diaphragm. T4b tumors are unresectable cancers invading other adjacent structures, such as aorta, vertebral body, and trachea.

A regional lymph node has been redefined to include any paraesophageal node extending from cervical nodes to celiac nodes (Table 1). In classifying $\mathrm{N}$, the data support convenient coarse groupings of number of cancer-positive 
nodes $(0,1-2,3-6, \geq 7)$. These have been designated N0 (none), N1 (1-2), N2 (3-6), and N3 ( $\geq 7$ ) and are identical to gastric $\mathrm{N}$ classifications.

The subclassifications M1a and M1b have been eliminated, as has MX (Table 1). Distant metastases are simply designated M0, no distant metastasis, and M1, distant metastasis.

Nonanatomic classifications identified as important for stage grouping were histopathologic cell type, histologic grade, and tumor location (Table 1). The difference in survival between adenocarcinoma and squamous-cell carcinoma was best managed by separate stage groupings for stages I and II. Increasing histologic grade was associated with incrementally decreasing survival for early-stage cancers. For adenocarcinoma, the distinction of G1 and G2 (well and moderately differentiated) from G3 (poorly differentiated) was important for stage I and stage IIA cancers. For squamous-cell carcinoma, the distinction of G1 from G2 and G3 was important for stage I and II cancers. Tumor location (upper and middle thoracic vs. lower thoracic) was important for grouping T2-3N0M0 squamous-cell cancers.

\section{STAGE GROUPINGS}

Stage groupings and corresponding risk-adjusted survival curves are presented in Tables 2 and 3 and Figs. 1 and 2 , respectively.
TABLE 1 Summary of changes in anatomic classifications and additions of nonanatomic cancer characteristics

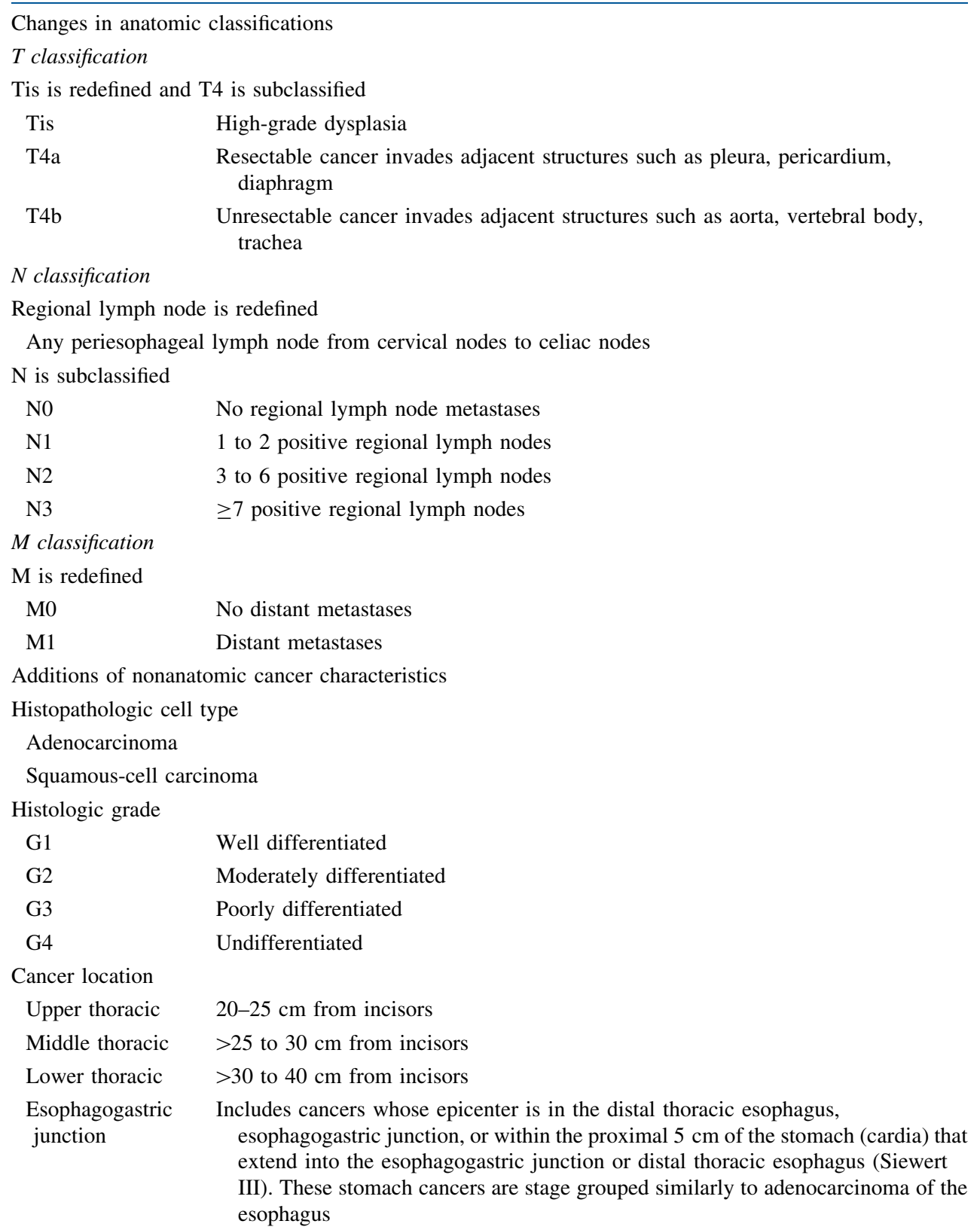


TABLE 2 Adenocarcinoma stage groupings

\begin{tabular}{lllll}
\hline Stage & T & N & M & G \\
\hline 0 & is (HGD) & 0 & 0 & 1 \\
IA & 1 & 0 & 0 & $1-2$ \\
IB & 1 & 0 & 0 & 3 \\
& 2 & 0 & 0 & $1-2$ \\
IIA & 2 & 0 & 0 & 3 \\
IIB & 3 & 0 & 0 & Any \\
& $1-2$ & 1 & 0 & Any \\
IIIA & $1-2$ & 2 & 0 & Any \\
& 3 & 1 & 0 & Any \\
& $4 a$ & 0 & 0 & Any \\
IIIB & 3 & 2 & 0 & Any \\
IIIC & $4 a$ & $1-2$ & 0 & Any \\
& $4 b$ & Any & 0 & Any \\
& Any & N3 & 0 & Any \\
IV & Any & Any & 1 & Any \\
& & & &
\end{tabular}

TABLE 3 Squamous-cell carcinoma stage groupings

\begin{tabular}{llllll}
\hline Stage & T & N & M & G & Location \\
\hline 0 & is (HGD) & 0 & 0 & 1 & Any \\
IA & 1 & 0 & 0 & 1 & Any \\
IB & 1 & 0 & 0 & $2-3$ & Any \\
& $2-3$ & 0 & 0 & 1 & Lower \\
IIA & $2-3$ & 0 & 0 & 1 & Upper, middle \\
& $2-3$ & 0 & 0 & $2-3$ & Lower \\
IIB & $2-3$ & 0 & 0 & $2-3$ & Upper, middle \\
& $1-2$ & 1 & 0 & Any & Any \\
IIIA & $1-2$ & 2 & 0 & Any & Any \\
& 3 & 1 & 0 & Any & Any \\
& $4 a$ & 0 & 0 & Any & Any \\
IIIB & 3 & 2 & 0 & Any & Any \\
IIIC & $4 a$ & $1-2$ & 0 & Any & Any \\
& $4 b$ & Any & 0 & Any & Any \\
& Any & N3 & 0 & Any & Any \\
IV & Any & Any & 1 & Any & Any \\
\hline
\end{tabular}

\section{ESOPHAGOGASTRIC JUNCTION CANCERS}

Besides being data driven, the 7th edition staging system harmonizes cancer staging across the esophagogastric junction. Previous staging produced different stage groupings for these cancers depending on use of either esophageal or gastric stage groupings. The 7 th edition staging system is for cancers of the esophagus and esophagogastric junction and includes cancer within the first $5 \mathrm{~cm}$ of the stomach that extend into the esophagogastric junction or distal thoracic esophagus (Siewert III).

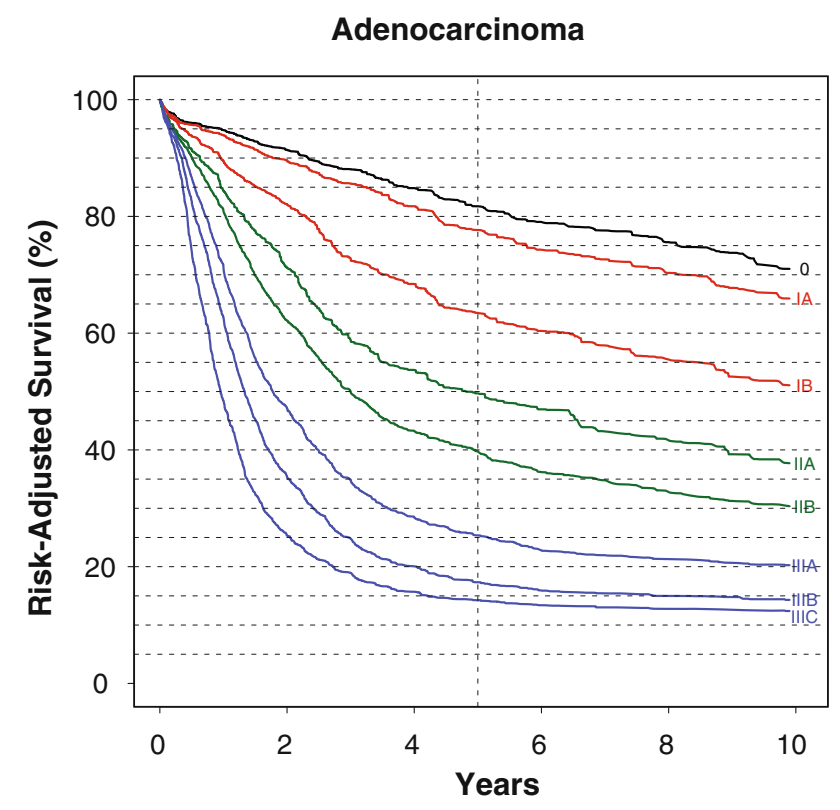

FIG. 1 Risk-adjusted survival for adenocarcinoma according to the American Joint Committee on Cancer Cancer Staging Manual, 7th edition, stage groups

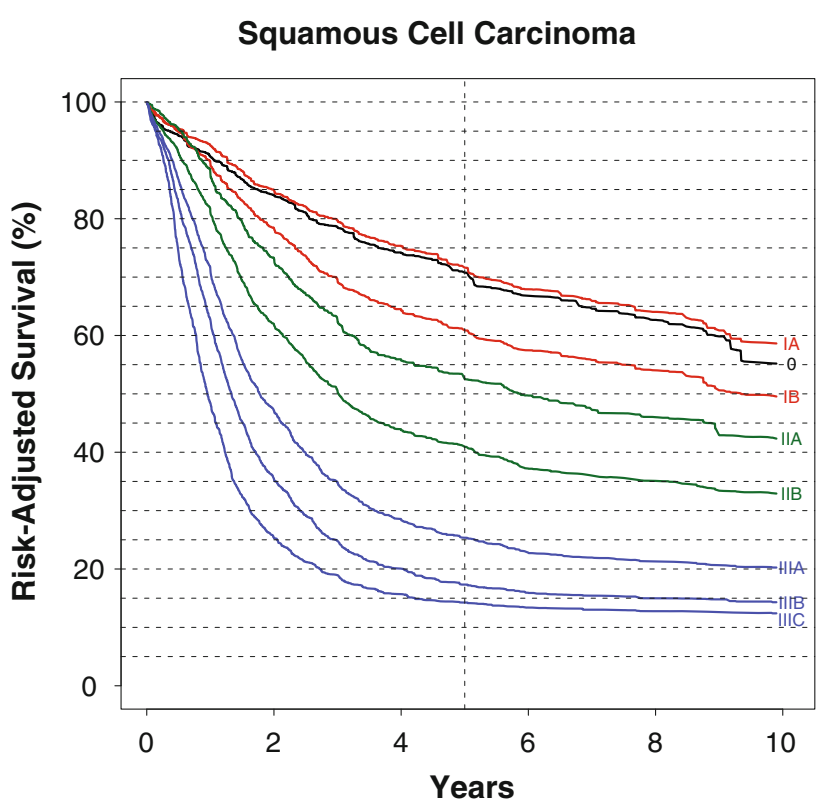

FIG. 2 Risk-adjusted survival for squamous-cell carcinoma according to the American Joint Committee on Cancer Cancer Staging Manual, 7th edition, stage groups

\section{CONCLUSIONS}

Previous stage groupings of esophageal cancer were based on a simple, orderly arrangement of increasing anatomic $\mathrm{T}$, then $\mathrm{N}$, then $\mathrm{M}$ classifications. These groupings were not consistent with data or cancer biology. 
Explanations for discrepancies relate to the interplay among TNM classifications, histopathologic type, biologic activity of the tumor (histologic grade), and cancer location. In contrast, the 7 th edition staging system is data driven; it is based on a risk-adjusted RF analysis of worldwide data, and it accounts for interactions of anatomic and nonanatomic cancer characteristics.

\section{REFERENCES}

1. Edge SB, Byrd DR, Compton CC, et al., eds. AJCC Cancer Staging Manual. 7th ed. New York: Springer-Verlag; 2009. p. 103-15.
2. Rice TW, Rusch VW, Apperson-Hansen C, et al. Worldwide Esophageal Cancer Collaboration. Dis Esophagus. 2009;22:1-8.

3. Ishwaran H, Blackstone EH, Apperson-Hansen C, et al. A novel approach to cancer staging: application to esophageal cancer. Biostatistics. 2009;10:603-20.

4. Rice TW, Rusch VW, Ishwaran H, et al. Cancer of the esophagus and esophagogastric junction: data-driven staging for the 7th edition of the AJCC/UICC cancer staging manual. Cancer (in press). 